\title{
Primary Signet-ring Cell Adenocarcinoma of the Urinary Bladder That Invades the Pelvic Wall Treated with Partial Cystectomy and Adjuvant Chemotherapy: A Case Report and Review of the Literature
}

\author{
(1) Çetin Yeşilli1 , ๑ Ali Harlak² \\ ${ }^{1}$ Private Cihan Hospital, Clinic of Urology, Izmit, Turkey \\ 2 Private Cihan Hospital, Clinic of General Surgery, Izmit, Turkey
}

\begin{abstract}
Primary signet-ring cell carcinoma of the urinary bladder is an exceedingly rare entity associated with poor prognosis. Thus, it is essential to distinguish this carcinoma from gastrointestinal metastases, as different therapeutic strategies are often necessary. We report the case of a 23 year old female patient with primary signet-ring cell carcinoma of the bladder that invades the pelvic wall and treated with partial cystectomy and adjuvant chemotherapy. No metastatic spread or recurrence was detected in the patient after 12 months of follow-up. To our knowledge, this is the first youngest female patient reported in the English literature.

Keywords: Urinary bladder, adenocarcinoma, signet-ring cell carcinoma, partial cystectomy
\end{abstract}

\section{Introduction}

Primary signet-ring cell carcinoma (PSRCC) of the urinary bladder, which was first reported by Saphir in 1955 (1), is a rare variant of adenocarcinoma and comprises only $0.24 \%$ to $2 \%$ of all primary epithelial urinary bladder tumours. It is associated with a poor prognosis and is generally resistant to chemotherapy and radiotherapy $(2,3,4)$.

Herein, we report the case of 23 year old female patient with PSRCC of the urinary bladder that invades the pelvic wall and treated with partial cystectomy and adjuvant chemotherapy. To our knowledge, this is the second case and the first youngest female patient reported in the English literature.

\section{Case Presentation}

A 23 year old female patient presented with lower abdominal pain, dysuria, intermittent episodes of painless haematuria with amorphous clots of 3 months duration. The medical and familial histories were unremarkable. The general and abdominal physical examination, gynaecologic and breast examinations, gynecologic and breast examinations urine analysis and blood tests were normal. Ultrasound evaluations revealed a polypoidal vesical mass measuring $30 \times 32 \mathrm{~mm}$ arising from the posterolateral wall of the bladder dome. Both kidneys and upper urinary tracts appeared normal. Computed tomography (CT) with and without intravenous contrast administration demonstrated a solid lesion with a diameter of $3 \mathrm{~cm}$ leading to a filling defect in the dome of the bladder without distant metastasis (Figure 1a).

After clinical examination, the patient underwent a transurethral resection of the bladder tumour (TURBT) that revealed a white, calcified, solid, papillary tumour extending from the posterior bladder wall to the dome. Biopsy specimens were sent for histopathological examination.

Histological findings were consistent with poorly differentiated mixed mucinous and signet-ring cell adenocarcinoma. The tumour was seen infiltrating the underlying stroma and deep muscle (Figure 2a). Immunohistochemical studies demonstrated strong positivity for CK7 and CK20 (Figure 2b and 2c), and prostate-specific antigen was negative. Periodic acid-Schiff (PAS) stain showed intense pink strain of the cytoplasmic vacuoles of the tumour cells (Figure 2d). 


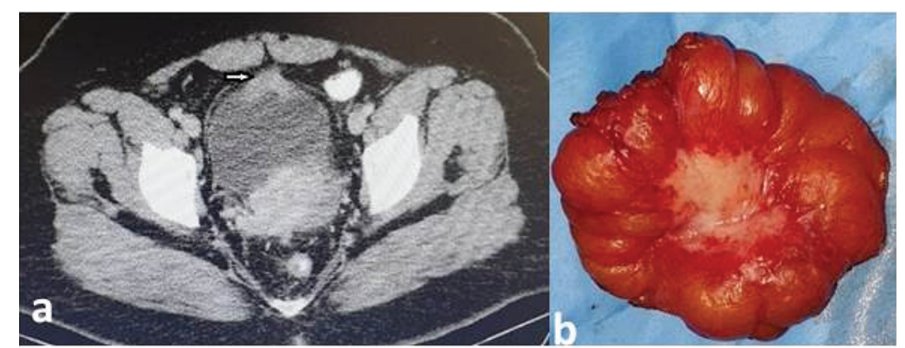

Figure 1. (a). Computed tomography image showing a solid lesion with a diameter of $3 \mathrm{~cm}$ leading to a filling defect in the dome of the bladder (arrow) without distant metastasis.

(b). Macroscopic appearance of partial cystectomy specimen

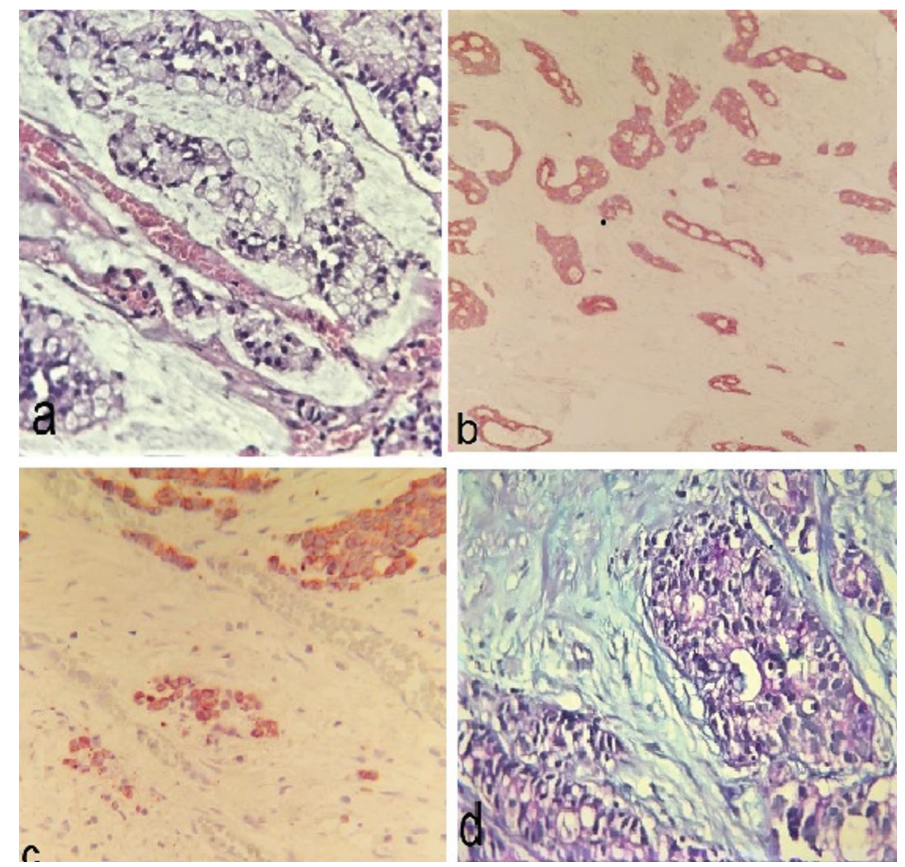

Figure 2. Histopathology of transurethral resection of the bladder tumour: poorly differentiated mixed mucinous and signet-ring cell adenocarcinoma (haematoxylin and eosin staining, $\times 200$ ) (a). Immunohistochemical studies demonstrated strong positivity for CK7 and (b) CK20 (c), and periodic acidSchiff staining showed intense pink strain of the cytoplasmic vacuoles of the tumour cells (d)

After this pathology report, gastrointestinal tract workup was carried out. Metastasis from a primary gastrointestinal tumour was considered, but gastroduodenoscopy and colonoscopy did not reveal a primary malignancy. Thorax CT and tumour markers such as alpha fetoprotein, cancer antigen (CA)-125, CA15-3, CA19-9 and carcinoembryonic antigen were normal. Fluorodeoxyglucose-positron emission tomography (FDG-PET) scan revealed a $30-\mathrm{mm}$ nodular lesion with increased FDG involvement in the bladder anterosuperior wall extending to the perivesical area. No pathologic FDG involvement was detected in other organs and lymph nodes. Radical cystectomy was recommended to the patient, but partial cystectomy was planned because the patient refused. Partial cystectomy and pelvic lymph node dissection were performed through a Phannenstiel incision. The peritoneum was opened, and generous margin of excision was achieved. No obvious pelvic lymphadenopathy was noted. Tumour metastasis was detected in the bilateral pelvic side wall and then resected. Pelvic lymphadenectomy to the bifurcation of the aorta was performed. The recovery following surgery was uneventful.

Macroscopic examination of the partial cystectomy specimen revealed an ulcerated mucosa, cut surface revealed a solid tumour measuring $30 \times 25 \times 20 \mathrm{~mm}$, infiltrating the bladder wall, and perivesical soft tissue (Figure $1 \mathrm{~b}$ ).

Histopathological evaluation revealed a primary bladder carcinoma composed of mucinous and signet-ring cell components, showing nests of columnar cells and signet-ring cells floating in pools of extracellular mucin. The columnar cells contained pleomorphic hyperchromatic nuclei. Signetring cells contained intracytoplasmic mucin (Figure 3a) and strongly positive for CDX-2 and PAS (Figure $3 b$ and Figure $3 c$ ). The epithelium was ulcerated and inflamed and was focally continuous with the tumour. The tumour involved muscularis propria. Lymphovascular and perineural invasion was positive and surgical margin was negative.

Pelvic lymph node metastasis was not observed, but metastasis was detected in the specimen of the pelvic side wall material bilaterally.

After 1 month, the patient was given eight cycles of gemcitabine/ cisplatin. CT and PET scans showed no pathologic evidence 3 months after the last cycle of chemotherapy. Cystoscopy was performed 6 and 12 months after the last cycle of chemotherapy, and no tumour was detected.

The patient provided written informed consent which includes the case details, operation and agreed to the publication of histopathological images.

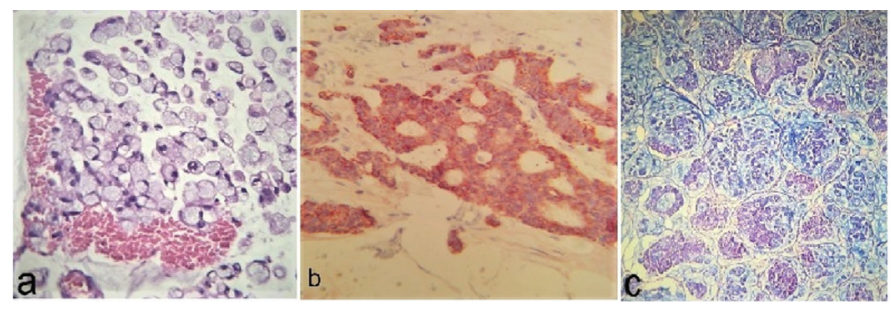

Figure 3. Histopathology of partial cystectomy. Multiple signet-ring cells (haematoxylin and eosin staining, $\times 200$ ) (a). Immunohistochemical studies demonstrated positive staining for CDX-2 (b) and periodic acid-Schiff staining (c)

\section{Discussion}

PSRCC of the bladder is a rare type of adenocarcinoma of the bladder. Most of the patients were in their seventh to eighth decades of life, there was male predominance and they were usually diagnosed at an advanced stage, usually demonstrating a subsequently poor prognosis (1).

To our knowledge, this is the youngest female patient reported in the English literature. An 18 year old male patient with PSRCC of the bladder treated with radical cystectomy was reported in 2017 (5).

Primary adenocarcinoma of the urinary bladder is derived from the urothelium that underwent glandular metaplasia, often 
Yeşilli and Harlak. Signet Ring Cell Adeno Carcinoma of the Bladder

in the context of chronic irritation of the vesical mucosa. The histologic diagnosis is based on the presence of characteristic signet-ring cells filled with cytoplasmic mucin-containing vacuoles compressing and displacing the nucleus into a peripheral crescent alongside the cell wall. The component of signet-ring cells in the tumour is variable, and it was reported as greater than $75 \%$ in almost half of the cases (6). In our patient, clinical imaging and immunohistochemistry were compatible with PSRCC of the bladder and the tumour tissue stained positive for CK7, CK20 and CDX-2.

Most adenocarcinomas of the urinary bladder result from direct extension from adjacent organs (e.g. colon and prostate). In a female patient, possible primary tumours include tumour from the colon, breast and genital system that should be considered differential diagnosis (7).

The variant signet-ring cell is poorly differentiated and is exceptionally described, and it is seen in approximately $0.24 \%$ of bladder cancers (8).

Clinical presentation of PSRCC of the bladder is similar to other bladder malignancies, and haematuria is the most common presenting symptom. Another rare presentation in the literature is mucinuria, which is reported in $3-12 \%$ of the patients (9). Our patient had a history of 3 months of intermittent painless haematuria with clot as the presenting symptom.

One of the main problems in cases of PSRCC of the bladder is to exclude metastatic adenocarcinoma from other sites of the body. It is essential to distinguish this carcinoma from metastases as different therapeutic strategies are often necessary. In our case, the gastrointestinal evaluation included esophagogastroduodenoscopy and colonoscopy, but no other tumour lesions were found.

SRCC is known to be associated with poor prognosis. On diagnosis, distant metastases are seen in approximately $25 \%$ of patients, and stage IV disease was found in almost half of the patients. This is due to the insidious progression of the disease (10). Its treatment is challenging, especially when diagnosed at an advanced stage. Surgery is the mainstay of treatment, and radical cystectomy is usually performed. Considering the rarity of this histologic type of tumour, there is no consensus regarding the management after surgical care. Chemotherapy and radiation therapy are discussed. Jayarajah et al. (11) reported a 71-year-old female patient with localised tumour treated with partial cystectomy. Wang and Wang (12) reported that 230 patients with pathologically confirmed PSRCC of the bladder were identified between 1973 and 2004. Overall, $26.5 \%$ presented with metastatic disease, $25.7 \%$ underwent TURBT only and $46.5 \%$ had partial or radical cystectomy. The 1-, 3 - and 10-year cancer-specific survival rates were 66.8, 40.6 and $25.8 \%$, respectively. Cystectomy was strongly associated with improved survival in patients with localised tumours that did not receive potentially curative cystectomy.

However, in our case, because the patient refused radical cystectomy, partial cystectomy and adjuvant chemotherapy (gemcitabine/cisplatin) was given following a deep TURBT. Follow-up cystoscopy, CT and PET scan at 6 and 12 months have shown no evidence of tumour recurrence. Partial cystectomy and adjuvant chemotherapy may be considered in selected young patients with stage IV disease who refused radical cystectomy.

\section{Conclusion}

PSRCC of the bladder is a rare tumour known to be associated with poor prognosis. However, partial cystectomy and adjuvant chemotherapy may be considered in selected young patients with stage IV disease without evidence of distant metastasis who cannot undergo radical cystectomy or refuse radical cystectomy.

\section{Acknowledgements}

Publication: The results of the study were not published in full or in part in form of abstracts.

Contribution: There is not any contributors who may not be listed as authors.

Conflict of Interest: No conflict of interest was declared by the authors.

Financial Disclosure: The authors declared that this study received no financial support.

\section{Ethics}

Informed Consent: The patient provided written informed consent which includes the case details, operation and agreed to the publication of histopathological images.

Peer-review: Externally peer-reviewed.

\section{Authorship Contributions}

Concept: Ç.Y., Design: Ç.Y., Data Collection or Processing: Ç.Y., Analysis or Interpretation: A.H., Literature Search: A.H., Writing: Ç.Y.

\section{References}

1. Saphir O. Signet-ring cell carcinoma of the urinary bladder. Am J Pathol 1955; 31:223-231.

2. Cobo-Dols $M$, Alcaide J, Gutiérrez V, et al. Primary signet-ring cell adenocarcinoma of the urinary bladder: a case report. Oncologia 2006;29:177-180.

3. El Ammari JE, Ahsaini M, Riyach $\mathrm{O}$, et al. Primary signet-ring cell carcinoma of the urinary bladder successfully managed with cisplatin and gemcitabine: a case report. J Med Case Rep 2013;7:37.

4. Michels ], Barbour S, Cavers D, et al. Metastatic signet-ring cell cancer of the bladder responding to chemotherapy with capecitabine: case report and review of literature. Can Urol Assoc J 2010;4:E55-7. doi: 10.5489/cuaj.831.

5. Allameh F, Karkan MF, Nilipour $Y$, et al. Primary signet-ring cell carcinoma of the urinary bladder successfully managed with radical cystectomy in a young patient. Case Rep Urol 2017;2017:9121078. doi: $10.1155 / 2017 / 9121078$.

6. Del Sordo R, Bellezza G, Colella R, et al. Primary signet-ring cell adenocarcinoma of the urinary bladder: a clinicopathologic and immunohistochemical study of 5 cases. Appl immunohistochem. Mol Morphol 2009;17:18-22.

7. Dadhania V, Czerniak B, Guo CC. Adenocarcinoma of the urinary bladder. Am J Clin Exp Urol 2015;3:51-63.

8. Sing J, Zherebitskiy V, Grynspan D, et al. Metastaticsignet-ring cell adenocarcinoma of the bladder: responsive to treatment? Can Urol Assoc J 2012;6:e15-9. doi: 10.5489/cuaj.11019. 
9. Nath V, Baliga M. Urinary baldder adenocarcinoma metastatic to the abdominal wall: Report of a case with cytohistologic correlation. Case Rep Pathol 2016;2016:8608412. doi: 10.1155/2016/8608412.

10. Akamatsu S, Takahashi $A$, Ito $M$, et al. Primary signet-ring cell adenocarcinoma of the urinary bladder. Urology 2010;75:615-618.

11. Jayarajah $\mathrm{U}$, Fernando $\mathrm{DMH}$, Herath $\mathrm{KB}$, et al. Primary signet-ring cell adenocarcinoma of the urinary bladder treated with partial cystectomy: a case report and review of the literature. Case Rep Urol 2017;2017:6829692. doi: 10.1155/2017/6829692.

12. Wang J, Wang FW. Clinical characteristics and outcomes of patients with primary signet-ring cell carcinoma of the urinary bladder. Urol Int 2011;86:453-460. 